\title{
De la obligación de confidencialidad en el arbitraje internacional y materias aledañas
}

Eduardo Silva

\section{Sumario}

1. Reflexión inicial. 2. De la confidencialidad implícita a la confidencialidad explícita en el arbitraje comercial. 2.1 Del derecho positivo de la confidencialidad. 2.2 De la confidencialidad como un problema de técnica contractual. 3. De la confidencialidad explícita en el arbitraje comercial internacional a la transparencia explicita en el arbitraje internacional de inversiones. 3.1 Fenomenología de la transparencia en el arbitraje internacional de inversiones. 3.1.1 De la transparencia como ausencia de confidencialidad.3.1.2 De la transparencia como confidencialidad limitada. 3.2 Los efectos perversos de la transparencia. 4. Conclusión.

\section{RefleXión INICIAL}

Es todavía más o menos común introducir cursos o libros sobre el arbitraje comercial internacional señalando las ventajas ${ }^{1}$

1. Cf., por cjemplo, E. GajlLard / J. Savage, Fouchard Gailland Goldman on International Commercial Arbitration, Kluwer Law Intemational, 1999. p. 1: "Intemational commercial arbitration has witnessed dramatic growth over the last twenty years. Although this reflects to a certain degree the underlying development of intemational commerce, international arbitration has flourished for a number of other reasons: arbitration is oflen perecived, rightly or wrongly, as being cheaper and less time-consuming than court proceedings, and is unquestionably more confidential; the resulting award is generally easicr to enforee than a court decision, largely thanks to the New York Convention; more importanily, international arbitration is now acknowledged -because its international character reflects the nature of the disputes being resolved - to be a neutral method of settling commercial disputes between parties from different nations, allowing each of the parties to avoid the "home" courts of its co-contractors; finally. international arbitration gives the parties substantial liberty to design their own dispute resolution me- 
de este, se dice, método alternativo ${ }^{2}$ de resolución de desavenencias. Quien escribe sobre el tema o enseña la materia del arbitraje comercial internacional tiende, por regla general, a promoverlo.

La palabra "alternativo" resaltada más arriba está, en principio, llena de significado. Bruno OpPETrT, en su artículo que acabo de citar, así lo sostiene. Las ventajas del arbitraje comercial internacional serían, en efecto, el polo opuesto de los que serían inconvenientes del proceso judicial adelantado ante cortes y jueces estatales. Así, por ejemplo, es todavía más o menos común escuchar o leer que el arbitraje comercial internacional es más rápido y menos costoso que ciertos procesos judiciales adelantados ante ciertas cortes nacionales; que el procedimiento del arbitraje comercial internacional es más flexible que él, también se dice, rígido procedimiento civil seguido por ciertos jueces y cortes (quien acude al arbitraje escoge, se afirma, ir donde el sastre; quien acude a las cortes, se agrega, escoge comprar su ropa en un supermercado); que el arbitraje comercial internacional es, por definición, más neutral que un proceso judicial nacional para resolver desavenencias comerciales internacionales (la premisa, cierta o errada, detrás de esta afirmación es que ninguna de las partes de una desavenencia confía en cortes y jueces del Estado de su contraparte); que en el arbitraje comercial internacional las partes escogen, con sus virtudes y defectos, a aquellos que van a dirimir su desavenencia (los árbitros) mientras que, en el sistema estatal

chanism, largely free of the constmints of national law. This party autonomy is found at every stage of the arbitral process and, although not of en fully exploited (with parties frequently preferring the plain application of institutional rules), is perhaps the most fundamental difference between international commercial arbitration and the courts. Indeed, it will generally be when parties make effective use of their entitlement to tailor their own arbitration proccedings to their needs that intemational arbitration will provide cheaper and more satisfactory justice than any national court system [...]".

2. Bruno Oppistr, por ejemplo, subraya que, "Selon une idée répondue, l'arbitrage commercial intemational offrirait une originalitê irréductible: ce que chercheraient les parties lorsqu'elles le mettent en cruvre, c'est à être jugées attrement qu'elles le scraicnt dans le cadre de tel ou tel autre mode de règlement de différends; par suitc, I'arbitre serait [citant l'cuuve de René David] « dans une situation qui diffère dans son principe de celle du juge ". Le recours à l'arbitmge par les acteurs du commeree intemational traduirait de leur part tant une volonté d'échapper à la fois à la compétence des juridictions ćtatiques et à l'cmprise des ordres juridiques nationaux qu'un désir d'une autre justice, administrée et rendue différemment; l'arbitrage commercial intemational affirmerait ainsi sa specificité non seulement à l'égard de la justice étatique, mais aussi vis-à-vis de l'arbitrage inteme et de tous les autres modes altematifs de résolution des litiges"; B. OrPetIT, "Philosophie de l'arbitrage du commerce international", Clunct, 1993, pp. 817.818, 
de administración de justicia, no es posible escoger a su juez; que los laudos arbitrales son más fácilmente ejecutables que las sentencias judiciales (gracias a la Convención de Nueva York sobre el reconocimiento y la ejecución de laudos arbitrales extranjeros del 10 de junio de 1958); y que el arbitraje comercial internacional es confidencial mientras que el proceso judicial ante cortes y jueces es público y, por ende, transparente.

Dichas ventajas, entre otras, siempre fueron, en nuestra opinión, afirmadas y reafirmadas sin demostración alguna. En el mejor de los escenarios, los miembros de la comunidad del arbitraje se pusieron tácitamente de acuerdo (se trataría del "contrato social" de la comunidad del arbitraje internacional), entre otras cosas, en que, para garantizar la supervivencia del arbitraje internacional, era necesario identificar sus ventajas y repetirlas, sin demostrarlas, sin cesar. Es sin duda por ello que, hoy dia, todas y cada una de las supuestas ventajas del arbitraje comercial internacional se hallan, sobre la base de la práctica real de dicha institución, bastante matizadas $y$, en el caso de algunas, controvertidas ${ }^{3}$. La virtud de la "confidencialidad" no es la excepción. Siempre, en efecto, se afirmó que el arbitraje comercial internacional era confidencial sin que, a fin de cuentas, se precisaran ni la fuente ni el ámbito de dicha confidencialidad. Dicha afirmación es hoy día fuente de controversias ${ }^{4}$.

Quizás el mejor esfuerzo que fuera realizado para proponer una fuente de la confidencialidad provino del derecho de las obligaciones y del contrato en general. Después de todo, el derecho arbitral, tal y como lo decía el Maestro Hinestrosa, no es sino una combinación (i) del derecho de las obligaciones y del contrato en general y (ii) de los principios de orden legislativo o, según el caso, jurisprudencial de la "autonomía del convenio arbitral" y de la "competencia-competencia"5. Nunca habrá mejor especialista en arbitraje internacional que quien domine, como el Maestro HinEstrosa, el derecho de las obligaciones y del contrato en ge-

3. Cf., por cjemplo, B. Oppetit, op. cit, pp. 821-824.

4. G. BORN, International Commerial Arhitration, Volume II, Wolters Kluwer, 2009, pp, 2250 y 2251.

5. Cf. E. Silva Romero, "El Contrato de Arbitraje", Legis, 2004. 
neral. Para algunos ${ }^{6}$, en efecto, la confidencialidad del arbitraje comercial internacional era y es un principio o elemento inherente al convenio arbitral internacional. Esta teoría siempre nos hizo pensar, en particular, en aquella disposición de ciertos códigos civiles en las cuales se definen los elementos esenciales, naturales y accidentales de los Contratos. En el Código Civil colombiano, por ejemplo, el artículo 1501 dispone lo siguiente:

\begin{abstract}
Elementos caracteristicos del contrato. Se distinguen en cada contrato las cosas que son de su esencia, las que son de su naturaleza, y las puramente accidentales. Son de la esencia de un contrato aquellas cosas sin las cuales, o no produce efecto alguno, o degenera en otro contrato diferente; son de la naturaleza de un contrato las que no siendo esenciales en él, se entienden pertenecerle, sin necesidad de una cláusula especial; $y$ son accidentales a un contrato aquellas que ni esencial ni naturalmente le pertenecen, y que se le agregan por medio de cláusulas especiales.
\end{abstract}

La confidencialidad del arbitraje comercial internacional sería, en este orden de ideas, un elemento natural del convenio arbitral internacional.

En cuanto al ámbito de la confidencialidad, por otra parte, tampoco era totalmente claro cuál era su órbita. ¿La confidencialidad, por ejemplo, cubría la existencia del arbitraje? ¿El laudo podía ser publicado sin el nombre de las partes? ¿En qué casos excepcionales, por otro lado, una de las partes del arbitraje comercial internacional podía dejar de cumplir su obligación de confidencialidad? ¿Cada vez, por ejemplo, que revelar aspectos del arbitraje internacional fuese necesario para la salvaguarda de sus derechos e intereses?

Quizás una de las raras delimitaciones de la noción de confidencialidad provenía, sin que las consecuencias jurídicas fueran claras, de la distinción que en ocasiones se hacía entre "confidencialidad" y "privacidad". A este respecto, por ejemplo, Gary BORN señala lo siguiente:

6. G. BORN, op. cit, p. 2280. 
It is important to distinguish between "privacy" and "confidentiality" in international arbitration. "Privacy" is typically used to refer to the fact that, under virtually all national arbitration statutes and institutional rules, only parties to the arbitration agreement -and not third parties- may attend arbitral hearings and otherwise participate in the arbitral proceedings. The privacy of the arbitration serves to prevent interference by third parties in the arbitral process (for example, by making submissions in the arbitration or by seeking to participate in the arbitral hearing), as well as to protect the parties' confidences against disclosure to third parties.

In contrast, "confidentiality" is typically used to refer to the parties" asserted obligations not to disclose information concerning the arbitration to third parties. Obligations of confidentiality extend not only to prohibiting the disclosure to third parties attending the arbitral hearings, but also to prohibiting the disclosture to third parties of hearing transcripts, as well as written pleadings and submissions in the arbitration, evidence adduced in the arbitration, materials produced during disclosure and the arbitral award(s). [...] [T] he confidentiality of the arbitral proceedings serves to centralize the parties' dispute in a single formm and to facilitate an objective, efficient and commercially-sensible resolution of the dispute, while also protecting the parties' confidences from disclosure to strangers?

No deja de ser comprensible, en todo caso, que siempre se haya insistido tanto en salvaguardar una confidencialidad implícita del arbitraje comercial internacional. Es evidente que la publicidad de los procedimientos puede afectar el "good will" de las partes y exacerbar la hostilidad entre ellas. Por una parte, parece evidente que ningún mercader internacional querrá que los socios potenciales con los que podría emprender operaciones económicas conjuntas ("hacer negocios") sepan que aquél, por ejemplo, no ha efectuado ciertos pagos. Por otra parte, parece también evidente que cualquier desavenencia puede agravarse en caso de ser ventilada públicamente. Como lo mencionaremos con más detalle más adelante, no hay nada más perverso que los efectos de los medios de comunicación sobre un litigio.

7. G. BoRN, op. cit, pp. 2251 y 2252. 
A pesar de las imprecisiones descritas más arriba en cuanto a su fuente y ámbito, la confidencialidad permaneció incólume por bastante tiempo. Ninguna felicidad, sin embargo, dura eternamente. Partes de arbitrajes comerciales internacionales decidieron llevar ante cortes estatales el debate de si su arbitraje era confidencial o no. Dichas cortes, en respuesta, sostuvieron que, para que el arbitraje fuera confidencial, era necesario que dicha obligación estuviese expresamente prevista en alguna parte. La confidencialidad, para ellas, devino un elemento accidental del convenio arbitral comercial internacional. De una confidencialidad implícita del arbitraje comercial internacional se transitó, en otras palabras, a una confidencialidad explícita del mismo (2).

Las cortes mencionadas, sin embargo, no han sido las únicas que han atacado aquella confidencialidad implícita del arbitraje comercial internacional. Como es de conocimiento público, el siglo XXI es la era de la ideología (entre otras) de la transparencia. Es así como en arbitrajes con partes estatales, especialmente en aquellos llamados "de inversión", se está, de alguna manera, invirtiendo el sentido de aquel principio implícito de confidencialidad del arbitraje. Hoy, más bien, parecería que, en dichos casos, el principio sería uno de "transparencia". De una confidencialidad explícita se está ahora transitando, en otros términos, a una transparencia implícita en ciertos casos (3).

\section{DE LA CONFIDENCIALIDAD IMPLÍCITA \\ A LA CONFIDENCIALIDAD EXPLICITA EN EL ARBITRAJE COMERCIAL INTERNACIONAL}

También es común leer en los más recientes textos sobre el arbitraje comercial internacional que la confidencialidad del arbitraje es un tema controvertido. En su tratado sobre la materia, GARY BORN precisa lo siguiente:

The confidentiality and privacy of international arbitration proceedings is a contentious and unsettled subject. A number of authorities 
regard confidentiality as an essential aspect of the arbitral process, which assists in the effective, efficient resolution of international disputes, and which must be given legal effect. At the same time, a substantial body of critics deny that confidentiality is a necessary or particularly beneficial feature of international arbitration proceedings, or that parties have a general legally-enforceable right to confidential arbitral proceedings $[\ldots]^{8}$.

Existiría, en este sentido, cierta tensión entre aquellos que, a pesar de la evolución del derecho positivo al respecto en ciertas latitudes, insisten en que el arbitraje comercial internacional es implícitamente confidencial (elemento natural) y aquellos que, a fin de cuentas, afirman que dicha confidencialidad implícita no observa ninguna fuente clara en el derecho positivo (2.1) y debe ser pactada expresamente para existir jurídicamente (elemento accidental) (2.2).

\subsection{Del derecho positivo de la confidencialidad}

Es, primero, importante señalar que ninguno de los tratados internacionales en materia de arbitraje trata el tema de la confidencialidad del procedimiento. Ni la Convención de Nueva York, ni la Convención de Ginebra, ni la Convención de Panamá comprenden normas al respecto. La conclusión es evidente: la comunidad internacional no ha logrado llegar a un consenso sobre el particular.

Es, por consiguiente, necesario acudir a los diferentes derechos nacionales que tienden a ser escogidos con mayor frecuencia como lex arbitri en búsqueda de un régimen que regule la materia. Ninguna de las legislaciones en materia de arbitraje de los Estados más comúnmente escogidos como sede de arbitrajes comerciales internacionales (Francia, Suiza, Reino Unido, etc.) comprende disposiciones expresas sobre la confidencialidad en el plano internacional. Hasta donde sabemos, en efecto, solamente el derecho arbitral peruano comprende una disposición re-

8. G. BORN, op.cit., pp. 2249 y 2250 . 
lativa a la confidencialidad del procedimiento arbitral. El artículo 51 de la Ley Peruana de Arbitraje, en efecto, dispone lo que sigue:

Artículo 51.- Confidencialidad.

1. Salvo pacto en contrario, el tribunal arbitral, el secretario, Ia institución arbitral y, en su caso, los testigos, peritos y cualquier otro que intervenga en las actuaciones arbitrales, están obligados a guardar confidencialidad sobre el curso de las mismas, incluido el laudo, asi como sobre cualquier información que conozcan a través de dichas actuaciones, bajo responsabilidad.

2. Este deber de confidencialidad también alcanza a las partes, suls representantes y asesores legales, salvo cuando por exigencia legal sea necesario hacer público las actuaciones o, en su caso, el laudo para proteger o hacer cumplir tun derecho o para interponer el recurso de anulación o ejecutar el laudo en sede judicial.

3. En todos los arbitrajes regidos por este Decreto Legislativo en los que interviene el Estado peruano como parte, las actuaciones arbitrales estarán sujetas a confidencialidad y el laudo será público, una vez terminadas las actuaciones.

La disposición peruana transcrita deja en claro, por el solo hecho de existir en dicha legislación, que un arbitraje comercial internacional con sede en Perú sería implícitamente confidencial salvo, claro está, pacto en contrario.

Dicha confidencialidad, sin embargo, no podría ser calificada de "elemento natural" del convenio arbitral internacional. Si algo debe resultar claramente de dicha disposición es que la confidencialidad no es una calidad inherente de todo arbitraje comercial internacional con sede en Perú sino una garantía que el legislador peruano ha decidido darle a éste.

El nuevo derecho arbitral francés merece una mención especial. Antes de la entrada en vigencia en mayo de 2011 de las modificaciones introducidas por el Decreto No. 2011-48 al Código de Procedimiento Civil francés, existía una cierta confidencialidad implícita del arbitraje comercial internacional. Hoy día, parecería claro que el arbitraje interno es implícitamente 
confidencial en Francia (por voluntad del legislador) y que el arbitraje internacional, por el contrario, no lo es. En efecto, como lo sostienen, por ejemplo, Emmanuel GaILLARD y Pierre de LAPASSE:

Le principe selon lequel l'arbitrage est confidentiel, sauf volonté contraire des parties (art. 1464, al. 4) ; en matière d'arbitrage relatif à la protection des investissements, un fort souci de transparence voit en effet le jour et il n'a dès lors pas été jugé opportun de prévoir un tel principe en matière d'arbitrage international. Cela signifie concrètement qu'en matière internationale les parties qui désirent bénéficier d'un régime de confidentialité devront le préciser expressément dans la convention d'arbitrage $e^{9}$.

Una importante razón por la cual la mayoría de los derechos arbitrales del mundo no tratan el tema de la confidencialidad puede ser que muchos de ellos se han inspirado de la Ley Modelo de la CNUDMI. La Ley Modelo de la CNUDMI, en efecto, no contiene ninguna disposición relativa a la problemática de la confidencialidad. Al decidir expresamente no tratar dicho tema, los redactores de la Ley Modelo de la CNUDMI afirmaron lo siguiente:

It may be doubted whether the Model Lnw should deal with the question whether an award may be published. Although it is controversial since there are good reasons for and against such publication, the decision may be left to the parties or the arbitration rules chosen by them ${ }^{10}$.

La CNUDMI, sin embargo, ha emprendido, a petición de varios Estados, la elaboración de un Anexo a su Reglamento de Arbitraje sobre el tema de la "transparencia" del arbitraje internacional. No es claro para nosotros, en este momento, cuál será la suerte de dicho documento.

9. E. Gailiard / P. DE LAPASSE, "Le nouveau droit français de l'arbitrage inteme et intemational", Dalloz, 2011, p. 188.

10. Cf. Report of the Secretary-General on Possible Features of a Model Law on International Commercial Arbitration, UN Doc. A/CN.9/207, I 17, XII Y.B. UNCITRAL 75, 90 (1981). 
Ha sido, en últimas, la jurisprudencia la que ha tratado con detalle el tema de la confidencialidad del arbitraje. Es en ella en donde encontramos la controversia a la que hace referencia Gary BORN. En este orden de ideas, en efecto, algunas cortes consideran que el convenio arbitral comprende implícitamente una obligación de confidencialidad mientras que otras, por el contrario, han sostenido que, para que el arbitraje sea confidencial, es necesario que dicha obligación haya sido pactada expresamente por las partes.

Las cortes que consideran que el convenio arbitral internacional comprende una obligación implícita de confidencialidad son, entre otras, las de Francia (antes del derecho arbitral de mayo de 2011) ${ }^{11}$, Inglaterra ${ }^{12}$ y Singapur ${ }^{13}$. No sobra resaltar que París y Londres son dos de las sedes más frecuentemente escogidas por las partes para sus arbitrajes comerciales internacionales ${ }^{14}$.

Por otra parte, las cortes de Australia ${ }^{15}$, Estados Unidos ${ }^{16}$, Suecia $^{17}$ y Suiza ${ }^{18}$ no reconocen la existencia de una obligación implícita de confidencialidad. Fueron, en especial, casos en Australia y Suecia los que pusieron en tela de juicio aquella afirmación sin demostración según la cual del convenio de arbitraje internacional surge una obligación implícita de confidencialidad.

El muy sonado caso australiano (decidido por la High Court of Australia) de 1995 opuso Esso Australia Resources a Plow$\operatorname{man}^{19}$. Gary BORN resume este caso de la siguiente manera:

11. Aïa c. Ojjeh, Corte de Apelaciones de Paris, Decisión del 18 de febrero de 1986, Rev: arb., 1986, pp. $586 \mathrm{ss}$.

12. Hassneh Ins. Co. of Israel c. Mew; Q.B., [1993] 2 Lloyd's Rep. 243; Cr. tambièn: Ali Shipping Corp. c. Shipyard Trogir [1998], English Court of Appcal, 2 All E. R. pp. 136 y ss.; y Oxford Shipping Co. Litd. c. Nippon liusen Kuisha, The Eastern Saga, Q.B, [1984] 3 All E.R. pp. 835 ss.

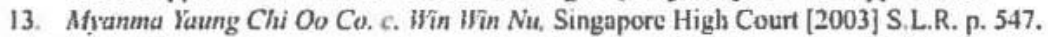

14. White \& Casc y Quecn Mary University of London, "2010 Intemational Arbitration Survey: Choices in International Arbitration", p. 19, http://www arbitrationonline org/docs/2010 InternationalArbitrationSurveyReport pdf (consultado el $2 !$ de septembre de 2012) - Londres $(30 \%)$, Geneva (9\%) y Paris y Tokio (7\%).

15. Esso Australi Resumes Lud, c. Plowman, 1995, Australian High Court, XX1 YBCA, p. 137.

16. Estades Unidos c. Panhandle Eastern Corp., 1988 C.D. Cal, I18 F.R.D. p. 346.

17. Bulgarian Foreign Trude Bank Lid. c. A.I.Trade Fin. Inc., Decisión del 27 de octubre de 2000, Swedish Supreme Court XXV1 YBCA, 2001, p. 291.

18. Decisión del 19 de junio de 2006, Swiss Fedcral Tribunal, 24 ASA Bull, 2006, p. 761.

19. Esso Australi Resources Lud. c. Plowman, 1995, op. cit. 
[...] The Esso Australin case presented the question whether one party to an arbitration (in this case, a government utility) could disclose documents produced in the arbitration by the adverse party (specifically, commercially-sensitive information regarding profit margins, petroleum reserves and production costs) to a governmental regulator. The Australian High Court permitted the disclosure, expressly refusing to recognize an implied obligntion of confidentiality in an Australian-seated international arbitration on the grounds that confidentiality was not "an essential attribute" of Australian arbitrations nor a necessary aspect of an arbitration agreement governed by Australian law $[\ldots]^{20}$.

La decisión Esso Australia, a pesar de negar la existencia de la confidencialidad implícita, delimitó indirectamente su ámbito. En efecto, dicha decisión sostuvo expresamente, en el sentido de lo descrito más arriba, que el arbitraje, aunque era "privado", no era "confidencial".

Por otro lado, el también muy sonado caso sueco que hizo temblar a la confidencialidad del arbitraje comercial internacional fue decidido por la Corte Suprema sueca el 27 de octubre de 2000 en el caso Bulgarian Foreign Trade Bank Ltd. contra A.I. Trade Fin. Inc. ${ }^{21}$. En este asunto, tal y como lo hizo la High Court of Australia en el caso Esso Australia, la Corte Suprema sueca rechazó la existencia de una obligación implícita resultante de todo convenio arbitral internacional. En resumen, la Corte Suprema sueca sostuvo lo siguiente:

A party to arbitration proceedings cannot be deemed to be bound by a duty of confidentiality, unless the parties have concluded an agreement concerning this ${ }^{22}$.

Las dos decisiones citadas más arriba dieron lugar a que la Comisión de Arbitraje de la CCI decidiera organizar una Task Force para analizar el tema de la confidencialidad del arbitraje comercial internacional. Luego de arduos trabajos y de notar las

20. G. Bork, op cit., p. 2262

21. Bulgarian Forcign Trade Bank Lid. c. A.I.Trade Fin. Inc., op. cit.

22. Ibid, p. 298. 
diferencias sobre el tema en jurisprudencias y doctrinas, la Comisión, en especial, recomendó no incluir una cláusula de confidencialidad en el Reglamento de Arbitraje de la CCI, y dejar que este tema fuera regulado por la voluntad de las partes y las leyes nacionales en materia de arbitraje $\mathrm{e}^{23}$.

Otros reglamentos de arbitraje, por el contrario, sí comprenden una cláusula de confidencialidad que las partes incorporan en su convenio arbitral por referencia al escoger uno de tales reglamentos como conjunto de normas aplicables al procedimiento arbitral. Este es el caso, por ejemplo, de los reglamentos de la SCC, JCAA, LCIA y Suizo del Arbitraje Internacional. El artículo 30.1 del Reglamento de Arbitraje de la LCIA, por ejemplo, dispone lo siguiente:

Unless the parties expressly agree in writing to the contrary, the parties undertake as a general principle to keep confidential all awards in their arbitration, together with all materials in the proceedings created for the purpose of the arbitration and all other documents produced by mother party in the proceedings not otherwise in the public domain - save and to the extent that disclosure may be required of a party by legal duty, to protect or pursue a legal right or to enforce or challenge an award in bona fide legal proceedings before a state court or other judicial authority.

Sobre la base de todo lo dicho anteriormente, es menester concluir que la confidencialidad del arbitraje comercial internacional es hoy día un problema de técnica contractual. Le corresponderá a las partes y a sus abogados, en efecto, pensar en el tema de la confidencialidad de su arbitraje comercial internacional al redactar el contrato respectivo y su cláusula compromisoria.

23. Reporte de la Comisión de Arbitraje de la CCI sobre el tema de la confidencialidad del arbitraje comercial internacional (no publicado). 


\subsection{De la confidencialidad como un problema de técnica contractual}

Así las cosas, las partes y sus abogados, por una parte, pueden prever la confidencialidad de su arbitraje comercial internacional de 3 maneras distintas:

- Es posible, en primer ligar, pactar expresamente la confidencialidad del arbitraje comercial internacional en el texto de la cláusula compromisoria correspondiente. En un texto reciente $^{24}$, la Comisión de Arbitraje de la CCI recomienda a las partes en arbitrajes con partes estatales que el siguiente texto sea considerado para pactar dicha confidencialidad:

Unless otherwise provided under the applicable law, ICCarbitration is not confidential per se. To protect confidentiality, states/state entities and their private contractual counterparties may therefore wish to modify the standard ICC arbitration clause as follows:

All disputes arising out of or in connection with the present contract shall be finally settled under the Rules of Arbitration of the International Chamber of Commerce by one or more arbitrators appointed in accordance with the snid Rules. The parties agree to keep confidential the existence of the arbitration, the arbitral proceedings, the submissions made by the parties and the decisions made by the arbitral tribunal, including its awards, except as required by applicable law and to the extent not alrendy in the public domain.

- Es posible, en segundo lugar, hacer referencia en el convenio arbitral a alguno de los reglamentos institucionales de arbitraje citados más arriba que prevén expresamente que el arbitraje descrito en sus disposiciones es confidencial (incorporación de la obligación de confidencialidad del arbitraje por referencia); $y$

24. Report of the ICC Commission on Arbitration Task Force on Arbitration Involving States or State Entities, "Arbitration Involving States and State Entities under the 1CC Rules of Arbitration", 2012, http://wwwicedrl com/? AUTH= (consultado el 21 de septicmbre de 2012). 
- Es posible, por último, estipular en el convenio arbitral que la sede del arbitraje comercial internacional será localizada en uno de aquellos Estados en los que la legislación (Perú) o la jurisprudencia (Francia -antes de mayo de 2011-, Inglaterra y Singapur) consagran una obligación implícita de confidencialidad.

Ahora bien: salvo que, según la primera modalidad descrita más arriba, el ámbito de la obligación de confidencialidad sea precisada, la referencia a un reglamento de arbitraje o a una lex arbitri que comprenda una obligación de confidencialidad puede dar lugar a disputas entre las partes en cuanto a su alcance. Dada la dilución de la confidencialidad en el arbitraje comercial internacional, parece ser cada vez más frecuente, en efecto, la situación en que una de las partes solicita expresamente al tribunal arbitral que éste ordene el respeto de la confidencialidad durante el procedimiento arbitral. Si, sobre la base de lo ya dicho, la fuente de la confidencialidad puede ser clara, su ámbito o alcance no se halla siempre definido. Por lo mismo, los árbitros internacionales han desarrollado una práctica según la cual éstos invitan a las partes del arbitraje a suscribir un acuerdo de confidencialidad preparado por aquéllos durante la instancia arbitral con el fin de preservarla.

Las partes y sus abogados, por otra parte, pueden preferir que el arbitraje no sea confidencial. De lo ya explicado resulta que dicha finalidad puede ser alcanzada a través de dos maneras distintas:

- En primer lugar, las partes podrían pactar explícitamente que su arbitraje debe ser "transparente" o que no debe ser "confidencialidad"; el problema al respecto será determinar en qué consiste un arbitraje "transparente" o "no confidencial". En el mismo texto citado más arriba, la Comisión de Arbitraje de la CCI, respecto del arbitraje con partes estatales, recomienda lo que sigue: 
[...] states/state entities and their private contractual counterparties can agree on greater transparency, for example by providing for the award, proceedings or submissions of the parties to be made public. It should be noted that the agreed degree of confidentiality or transparency can be changed in the course of the proceedings $[\ldots]^{25} ; y$

- Por otro lado, las partes pueden pactar la transparencia implícitamente al no tratar el tema y fijar la sede del arbitraje comercial internacional en un Estado cuyo derecho arbitral haya precisado que el convenio arbitral no produce (implícitamente) una obligación de confidencialidad. Este sería el caso, por ejemplo, de Australia, Estados Unidos, Francia (desde mayo de 2011), Suecia y Suiza.

Todo lo anterior se aplica al arbitraje comercial internacional. Una tercera vía para garantizar, sin pactarla, la no confidencialidad del arbitraje, ha surgido en los asuntos que involucran a un Estado soberano y que pueden ser calificados de arbitrajes de inversiones; en estos asuntos, en efecto, estaría surgiendo una tendencia a consagrar una "transparencia" implícita.

\section{DE LA CONFIDENCIALIDAD EXPLICITA EN EL ARBITRAJE COMERCIAL INTERNACIONAL A LA TRANSPARENCIA IMPLÍCITA EN EL ARBITRAJE INTERNACIONAL DE INVERSIONES}

El interés público involucrado en ciertos asuntos (pensemos, por ejemplo, en temas de salud pública y de medio ambiente) ha hecho, se dice, que los ciudadanos y el público en general tengan interés en conocer lo que está ocurriendo en un arbitraje internacional en el que su Estado es demandado.

25. Report of the ICC Commission on Arbitration Task Force on Arbitration Involving States or State Entitics, op. cit. 
Dicho interés se ha visto traducido, especialmente por algunas $\mathrm{ONGs}^{26}$ (cuyas orientaciones ideológicas son más o menos conocidas del público), en el deseo, rápidamente calificado de "derecho", de que los procedimientos arbitrales internacionales que involucren al Estado y conciernan temas de interés público sean "transparentes". Algunos Estados, entre los que se destacan Canadá y los Estados Unidos, han hecho también suya la "lucha por la transparencia"27. Quienes reclaman dicha "transparencia", sin embargo, no proponen ninguna definición de este término.

Las palabras "transparencia", "transparentar" y "transparente", que todo el mundo utiliza pero de las que pocos conocen el verdadero significado, son definidas por el Diccionario de Castellano de la Real Academia de la Lengua Española como sigue:

transparencia. f. Cualidad de transparente. // 2. Lómina transparente que contiene dibujos o textos y a la que se pueden aĩadir datos durante su proyección. // 3. Cinem. Proyección sobre una pantalla transparente de imágentes móviles filmadas con antelación, que sirve de fondo a una acción real.

transparentar. tr. dicho de un cuerpo: permitir que se vea o perciba algo a través de él. // 2. Dicho de un cuerpo: Ser transparente. U.t.c prnl. // 3. Prnl. Dicho de una cosa que no se manifesta o declarn: Dejarse descubrir o adivinar en lo patente o declarado. Transparentarse un propósito, el temor, la alegría U.t.c.tr. // 4. coloq. Dicho de una prenda de ropa: Estar demasiado fina por el desgaste. //5. coloq. Dicho de una persona: Estar demasiado flaca.

transparente. (De lat. Trans-, a través, y parens, -entis, que aparece). adj Dicho de un cuerpo: A través del cual pueden verse los ob-

26. Por ejemplo. World Wildlife Fund (WWF) y Centre for International Enviromental Law (CIEL).

27. Cf. Declaración de la Comisión de Libre Comercio sobre la participación de una tercera parte, del ? de octobre de 2003, http://www.international ge.ca/trade-agreements-accords-commerciaux/assets pdffs/nondisputing-fr.pdf (consultado el 21 de septiembre de 2012); Declaración del Canadá, butp://www.international ge.ca/trade-agreements-accords-commerciaux/disp-diff/nafta-transparencyalena-transparcnce aspx? lang= fra\&vicw=d (consultado el 21 de scptiembre de 2012); Cf, tambićn con relación a los Estados Unidos, G. BorN, op. cit, p. 2274. 
jetos claramente. // 2. Dicho de un cuerpo: translúcido. // 3. Que se deja adivinar o vishumbrar sin declararse o manifestarse. // 4. Claro, evidente, que se comprende sin duda ni ambigiiedad // 5. m. tela 0 papel que, colocado a modo de cortina delante del hueco de ventanas o balcones, sirve para templar la luz, o ante una luz artificial, sirve para mitignrln o para hacer aparecer en él figuras o letreros. //6. Ventana de cristales que ilumina y adorna el fondo de un altar $(\ldots)^{28}$.

De las definiciones transcritas resulta, antes que nada, que dichos términos han sido insertados en el lenguaje ordinario para calificar objetos del mundo tangible. Así, por ejemplo, las frases "el agua del riachuelo es transparente" o "la transparencia del vidrio es admirable" tienen un sentido inequívoco para nosotros. La frase "el arbitraje de inversiones debe ser transparente", por el contrario, es, por decir lo menos, ambigua. Al haber sido transpuesta sin mayores explicaciones del lenguaje del mundo tangible al del mundo intangible, la palabra "transparente" en la última frase no puede cumplir sino, a lo sumo, una función metafórica.

¿Qué quiere significar la metáfora de la "transparencia" en el mundo intangible y, en especial, en el contexto de un arbitraje internacional de inversiones? No es claro para nosotros que, hasta este momento, sea absolutamente claro lo que la palabra "transparencia" pretende designar en el arbitraje internacional de inversión. Es, por ende, necesario comenzar por presentar la fenomenología de la transparencia en el arbitraje internacional de las inversiones (3.1). Dicha descripción nos enseñará, a su turno, que la "transparencia" produce varios efectos perversos indeseables (3.2).

\subsection{Fenomenología de la transparencia en el arbitraje internacional de inversiones}

Es quizás posible definir la "transparencia", así sea de forma transitoria, como la ausencia de confidencialidad (3.1.1) o la con-

28. Diccionario de la Lengua Española, 22² cd., Real Academia Española, 2001, p. 2213. 
fidencialidad limitada (que equivale a una transparencia limitada) (3.1.2).

\subsubsection{De la transparencia como ausencia de confidencialidad}

Según el deseo de quienes reclaman transparencia y la práctica reciente de tribunales arbitrales internacionales de inversión, la transparencia, según su primera acepción de ausencia de confidencialidad, observaría dos manifestaciones.

En primer lugar, la transparencia daría un derecho a terceros, ciudadanos o público en general, a conocer lo que está ocurriendo en un arbitraje internacional de inversiones en el que un Estado es demandado y que concierne el interés público. Este derecho puede ser ejercido, nos parece, durante el arbitraje y después del mismo. Durante el arbitraje, el derecho de conocimiento, según lo que se puede leer hoy día en tratados ${ }^{29}$, reglamentos de arbitraje $^{30}$, decisiones arbitrales ${ }^{31}$ y el proyecto de anexo sobre la confidencialidad de la $\mathrm{CNUDMI}^{32}$, concierne la existencia del arbitraje, la posibilidad de leer los documentos intercambiados durante la instancia arbitral y la posibilidad de asistir a las audiencias. Después del arbitraje, las mismas fuentes señaladas ${ }^{33}$ indican que las partes tienen un derecho a la publicación del laudo arbitral.

29. Por ejemplo, las interpretaciones dadas a los Tratados CIADI y TLCAN; BIT modelo de los Estados Unidos de 2004, articulos 29-1 y 29.2; Tratado de Libre Comercio entre Estados Unidos, Centroamérica y República Dominicana, articulos 10-21-1 y 10.21.2, B1T Modelo elaborado por el Canadả de 2004. articulo 38-3.

30. Reglas de Arbitraje del CIADI. Regla 32-2; Reglamento Administrativo y Financicro del CIADI. Regulación 22.1.

31. Por cjemplo, Amco Asia Corporation et al, c. República de Indonesia, Caso CIADI No. ARB/81/1, Decisión del 9 de diciembre de 1983.

32. CNUDMI, Grupo de Trabajo II (Arbitraje y Conciliación), "Solución de Controversias Comereiales Preparación de una Norma Juridica sobre la Transparencia en los Asbitrajes entre Inversionistas y un Estado". 57 periodo de sesiones Viena, I a 5 de octubre de 2012 , http://daccess-ddsny.un.org/doc/UNDOC/LTD/V12/552/50/PDF/V1255250 pd?? OpenElement, (consultado el 21 de septiembre de 2012), proyecto de artículos 3.1, 3.2 y 7.

33. Reglas de Arbitraje del CIADI. Regla 48-4; CNUDMI, Grupo de Trabajo Il (Arbitraje y Conciliación), op. cit; articulo 4; Tratado de Libre Comercio entre Estados Unidos, Centroamérica y República Dominicana, 10.21.1(c) y 29.1; BIT Modelo elaborado por el Canadá de 2004, artículo 38-3. 
Cuando se habla de transparencia, mucho se insiste en las bondades de la publicación de los laudos arbitrales proferidos en arbitrajes internacionales de inversión. Al respecto, dos sofismas son utilizados con alguna frecuencia. Por una parte, la publicidad de los laudos, se dice, contribuiría a la formación de una jurisprudencia arbitral. Todos sabemos, sin embargo, que la expresión jurisprudencia arbitral es un abuso de lenguaje. Por otra parte, se insiste, en el escenario de casos relacionados, en la necesidad de evitar el dictado de decisiones contradictorias. Este problema, sin embargo, no parece poder ser evitado mediante la publicación de los laudos sino a través de figuras procesales como la acumulación de procedimientos.

En segundo lugar, la transparencia daría un derecho a terceros de participar en el procedimiento arbitral. Se trata, en este punto, de la posibilidad de presentar memoriales y alegatos orales por parte de amicus curiae en el arbitraje internacional de inversiones ${ }^{34}$. La posibilidad de esta participación fue aceptada en un primer momento por los tribunales arbitrales ${ }^{35}$. Luego, dicha jurisprudencia fue plasmada en varios tratados internacionales ${ }^{36}$. Veamos dos ejemplos:

34. Cf. sobre el tema: B. STERN, "Un petit pas de plus: I'Installation de la Société Civile dans I'Arbitrage CIRDI entre Etat et Investisseur", Volume 2007(1), Rev arb,2007, pp. 3-43: "Transparence et Participation de Tierces Parties aux Procédures de Règlament des Diffërends entre Investisseurs et Elats", Déclaration du Comité d'Investissement de l'OCDE, junio 2005, www.ocde.org/investement: E. LEVINE, "Amicus curiae in Iniernational Investment Arbitration: The implications of an Increase in Third Party-Party Participation", Volume 29(1), Berkeley Journal of International Law, 2011; 1. MAxweLL, "Transparency in Investment Arbitration: Are Amici Curiae the Solution?", Volume 3(2), Kluwer Law International, 2007, pp. 176-186; N. BLACKABY / C. RICIARD, "Amicus Curiac, A Panacea for Legitimacy in investment Arbitration?" en M. KAUSHAL/A. Kaushiat/K. Chung / C. BALCIIN, The Blacklash Against Investment Arbitration, Kluwer Law International, 2010, pp. 253-274; J.A. VANDUZER, "Enhancing the Procedural Legitimacy of Investor-State Arbitration through Transparency and Amicus Curiae Participation". Volume 52, Revue de droit de MeGill, 2007; F. Grisel / E. VINUAl.Fs, "L'amicus Curiac dans l'Arbitrage d'Investissement", ICSID Review. Foreign Investment Journal, 2008, pp. 380 ss.

35. Methanex c. Estados Unidos, caso CNUDMI, Decisión del 15 de enero de 2001 y United Parcel Service c. Canada, caso CNUDMI, Decisión del 17 de octubre de 2001.

36. BIT modelo de los Estados Unidos de 2004, articulo 28,3, Tratado de Libre Comercio entre Estados Unidos, Centroamérica y República Dominicana, artículo 10.20.3: BIT Modelo elaborado por el Canadá de 2004, artículo 39; Acuerdo de libre comercio firmado el 6 de junio de 2003 entre los Gobiemos del Estados Unidos y Chile, artículo 10.19.3; Acuerdo de libre comercio firmado el is de junio de 2004 entre los Gobicmos del Estados Unidos y Marruecos, articulo 10.19.3: Acuerdo de libre comercio firmado el 6 de mayo de 2003 entre los Gobiemos del Estados Unidos y Singapur, articulo 15.19.3; Acucrdo de Inversión Internacional firmado entre los Gobiemos del Canadá y Perú, artículo 836 y anexo 836.1; Acuerdo de Inversión Internacional firmado entre los Gobiemos del Canadá y Jordania, articulo 39 y anexo 39 . 
Primero, en el caso UPS c. Canadáa ${ }^{37}$, dos sindicatos canadienses requirieron al Tribunal Arbitral su participación en la disputa bajo el fundamento que: (i) tendrían un interés directo en el caso; (ii) podrían traer al procedimiento una perspectiva diferente; y (iii) que su participación promovería la apertura del proceso arbitral. El Tribunal admitió la participación de los sindicatos canadienses en el caso para la presentación de memoriales escritos, aunque no haya permitido la participación de terceros como partes en el procedimiento.

Segundo, el BIT Modelo elaborado por el Canadá de 2004, en su artículo 39.1, establece:

Any non-disputing party that is a person of a Party, or has a significant presence in the territory of a Party, that woishes to file a written submission with a Tribunal (the "applicant") slall apply for leave from the Tribunal to file such a submission, in accordance with Annex C.39. The applicant shall attach the submission to the application.

Con todo, la transparencia referida en las fuentes citadas más arriba no parece, como veremos, ser absoluta.

\subsubsection{De la transparencia como confidencialidad limitada}

Un segundo sentido de la palabra "transparencia" designaría la necesidad de que el arbitraje de inversiones no fuera confidencial sino en algunos casos excepcionales. La transparencia, en este sentido, tendría dos límites principales.

Primero, los diferentes sistemas de arbitraje internacional utilizados en materia de inversión prevén una excepción a la transparencia cuando es necesario proteger información confidencial. Dicha información puede tratar, por ejemplo, secretos oficiales (como el secreto de defensa) y secretos de orden comercial. 
La regla 32.2 de las Reglas de Arbitraje del CIADI establece excepciones al procedimiento de publicidad para proteger información confidencial o privilegiada. Dicha protección también está presente en el artículo 29 del modelo de BIT de los Estados Unidos de 2004 y en el BIT modelo del Canadá de 2004 (artículo 38).

A su turno, las Reglas de la IBA sobre la Obtención de Pruebas en el Arbitraje Comercial Internacional prevén la protección de los documentos confidenciales, haciendo referencia explícita a los secretos de Estado ${ }^{38}$.

Segundo, parecería existir hoy día una tendencia jurisprudencial a limitar la transparencia en los arbitrajes internacionales de inversión cuando ésta supone que la integridad del procedimiento arbitral está en peligro. Varios tribunales arbitrales $\mathrm{CIADI}^{39}$ han, en efecto, emitido órdenes procesales destinadas a mantener el status quo y evitar que la desavenencia se agrave.

En el caso Biwater Gauff (Tanzania) Limited c. Tanzania, el Tribunal, al tiempo que reconoce el carácter público de la controversia, ponderó ser necesario limitar la publicidad del caso para asegurar el buen funcionamiento y la integridad del procedimiento. El fragmento de la decisión transcrito abajo es muy ilustrativo:

It is self-evident that the prosecution of a dispute in the media or in other public fora, or the uneven reporting and disclosure of documents or other parts of the record in parallel with a pending arbitration, may aggravate or exacerbate the dispute and may impact upon the integrity of the procedure. This is all the more so in very public cases, such as this one, zuhere issues of wider interest are raised, and where there is already substantial media coverage, some of which already being the subject of complaint by the parties. ${ }^{40}$

38. IBA Rules on the Taking of Evidenec in Intemational Commercial Arbitration, 1999, articulo 9.2(b)( $)$.

39. Amco dsia Corporation y otros c. Reptiblica da Indonesia caso CIADI No ARB 81.1, Decisión del 9 de diciembre de 1983; Metalclad Corp. C. Mérico, caso ClADI No. ARB(AF) 97/I, Decisión del 30 de agosto de 2000; Lowen Group Inc \& Raymond L. Lowen c. Estados Unidos, No. ARB(AF) 98/3, Decisión del 9 de enero de 2001; Biwrater Gauff (Tunzania) Limited c. Tunzania, caso CIADI No. ARB/05/22, Orden Procesai n. 3 del 29 de septiembre de 2006.

40. Bistuter Gauff (Tunzania) Limited c. Tanzania, op, cit . f 136. 
Esta segunda excepción al creciente principio de transparencia deja entrever sus efectos perversos.

\subsection{Los efectos perversos de la transparencia}

Quizás los efectos perversos de la transparencia provienen, como regla general, de su origen ideológico o, si se quiere, político.

La transparencia, en efecto, no ha sido introducida en el arbitraje internacional de inversiones para arreglar un problema de orden técnico-jurídico de dicho mecanismo de resolución de desavenencias. La transparencia, en efecto, no busca hacer que el arbitraje internacional de inversiones sea más eficaz. La transparencia, por el contrario, pretende, se dice, reducir un déficit democrático en el arbitraje de inversión ${ }^{41}$.

La premisa mayor de esta posición sería que un arbitraje secreto de cuestiones esenciales para un Estado puesto que conciernen sus valores más esenciales (llamados, en conjunto, "interés público") no sería un mecanismo legítimo de resolución de diferendos. La pregunta (en ocasiones retórica) que formulan los partidarios de la transparencia es la siguiente: ¿cómo es posible que la resolución de asuntos graves que conciernen el interés general sea secreta y no pueda ser conocida y supervisada por los ciudadanos y el público en general ( $^{42}$

41. Véase: N. Blackady, "Public Interest in Investment Treaty Arbitration" en A.J. Van den Berg (ed.), International Commercial Arbitration: Contemporary Questions, Volume 11. 1CCA Congress Series, London, 2002; Mabel I. Egonu, "Greater transparency' in the arbitration proces afford the opportumity. not only to be av'are that there is a dispute, but also to question the action of their governments"; $\mathrm{M}$. EgonU, "Investor-State Arbitration Under ICSID: A Case for Presumption Against Confidentiality?" JIA Volume 4(5), Kluwer Law International, 2007, pp. 479-489, 487.; Cindy Buys: "To the extent that meblic international arbitrations are made more transparent, denocratic ideals are cnhanced hecause the public has the opportunity to observe the process and hold the governments accountable for their action with respect in the arbitration and for the result", C. BuYs, "The Tensions between Confidentiality and Transparency in International Arbitration", 14 ARIA, 2003, p. 12I; C. RoGers, "Transparency in International Commercial Arbitration", Kansas Law Review, 2006; Bocconi Legal Studies Research Paper No. 06-10.

42. Véase: N. Bl.ACKABY, op. cit. 
Nosotros pensamos, sin embargo, que dicha transparencia, en últimas, puede ser perjudicial para el Estado y sus ciudadanos y criticable desde varios puntos de vista. Cuatro críticas podrían, en particular, ser hechas a la transparencia.

En primer lugar, es posible expresar serias dudas en cuanto a la teoría política detrás de las reivindicaciones de transparencia. Parecería, en efecto, que la concepción política de la transparencia supone una democracia popular altamente participativa. Los ciudadanos, según esta postura, deberían poder controlar todas las acciones emprendidas por el aparato estatal. ¿Es realmente necesario que los ciudadanos controlen todas las acciones de la Administración? ¿No deberían los ciudadanos y el público en general simplemente confiar en los representantes políticos que han elegido?

Lo que es, para nosotros, evidente al respecto es, en segundo lugar, que el control de los ciudadanos y del público en general que resulta de la transparencia puede convertirse en un obstáculo importante en el desarrollo de la estrategia que un Estado adopte en un arbitraje internacional de inversiones. La transparencia o publicidad se ha convertido en una nueva "variable" de la ecuación que inversionistas y Estados receptores de inversión deben considerar en la resolución de sus desavenencias. Cinco ejemplos pueden ilustrar esta crítica:

- el acceso a lo que ocurre en el arbitraje podría permitir a la oposición política del Estado demandado criticar (la mayoría de las veces con motivaciones partisanas) la estrategia adoptada por éste en el arbitraje; dichas críticas, realizadas en foros públicos o a través de los medios de comunicación, por lo general generan, en nuestra sociedad del espectáculo, reacciones de quienes regentan el poder; si tales reacciones, que son declaraciones públicas, no son coordinadas con el equipo de defensa del Estado demandado en el arbitraje, pueden causar perjuicios irreparables en cuanto a la estrategia adoptada en éste; 
- la publicación de memoriales y posiciones del Estado puede causar el retorno de la protección diplomática; el Estado del inversionista, en efecto, podría querer intervenir ante ciertas afirmaciones que el Estado demandado -receptor de la inversión- haga;

- la publicidad de los arbitrajes de inversión, además, podría llegar a disuadir inversores potenciales de invertir en el Estado demandado; la paradoja es evidente: un sistema jurídico creado, como lo establecen todos los tratados bilaterales de promoción y protección de inversiones, para promover la inversión extranjera, lograría a fin de cuentas que ésta, por lo menos, disminuyera;

- como ya lo mencionamos, la publicidad de los arbitrajes de inversión puede agravar la desavenencia entre las partes; un corolario de esta posible agravación merece ser subrayado; los funcionarios públicos que tengan la facultad de obligar a la Administración tendrán dificultades en celebrar una transacción del litigio cuando éste sea de conocimiento del público y las diferencias entre las partes, según lo ya dicho, se haya politizado; $y$

- la publicidad del arbitraje de inversiones puede acarrear problemas en materia probatoria; testigos potenciales pueden, en efecto, sentirse intimidados, y funcionarios públicos pueden ser más reacios al ser solicitados para compartir información y entregar documentos.

No sobra precisar que, si el objetivo primordial del arbitraje de inversión es despolitizar las controversias inversionista-Estado $^{43}$, la ideología de la transparencia entra necesariamente en colisión, dados los ejemplos descritos más arriba, con dicho objetivo.

43. C. SCHREuER. ICSID Comention: A Commentan; Cambridge University Press. 2009, pp. 416 I 4 y 419 ๆ 14. 
En tercer lugar, otro efecto perverso de la transparencia puede ser el aumento de los tiempos y costos del procedimiento. Sobre el particular, los críticos de la transparencia señalan, en particular, los plazos y expensas adicionales que la participación de amicus curiae puede causar en el arbitraje.

Lo ocurrido en el caso Glamis Gold ${ }^{4}$ ilustra bien esta crítica. En dicho caso, en efecto, el Tribunal aceptó, en una fase inicial, la intervención de un tercero interesado en la disputa, Quechan Indian ${ }^{45}$, bajo los argumentos de (i) que la participación de terceros estaba de acuerdo con la intención de las partes contratantes del TLCAN y (ii) que dicha participación no causaría dilaciones indebidas en el procedimiento. Sin embargo, más tarde, tres otras empresas ${ }^{46}$ solicitaron su participación en el procedimiento, al mismo tiempo que Quechan Indian también había solicitado que se presentase un nuevo memorial, totalizando, así, cinco memoriales en el mencionado procedimiento. Aunque el Tribunal haya limitado el número de páginas de esos documentos a no más de veinte, no se puede negar que la participación de terceros en calidad de amicus curine puede traer consecuencias negativas al procedimiento.

Por último, la ideología de la transparencia ha causado un deseo desenfrenado de legislar sobre la materia tanto al nivel de los Estados como al nivel de la CNUDMI. Nosotros pensamos, sin embargo, que regular todo lo relativo al arbitraje internacional (i) es una actitud contraria a la flexibilidad que hace su esencia y (ii) le resta a la institución su finalidad de ser, como ya lo explicamos, un mecanismo alternativo de resolución de desavenencias.

44. Glamir Gold. LId. c. Estados Unidos, Caso CNUDMI, en particular las Decisiones del 16 septiembre 2005 y del 10 de octubre de 2006.

45. Quechan Indian Nation of Fort Yuma Arizona and California USA.

46. Friends of the Earth Canada and Friends of the Earth United States, National Mining Association y Sicrra Club and Earthworks. 


\section{Conclusión}

¿Qué se puede decir en conclusión? ¿Que la confidencialidad del arbitraje comercial internacional es un mito (se trataría hoy día de un problema de técnica contractual) y que la transparencia del arbitraje internacional de inversiones proviene de una ideología ajena al interés de resolver litigios bien y rápido que ha puesto en riesgo las estrategias procesales de inversionistas y Estados? No nos resta, con todo, sino esperar que, ante mitos e ideologías (dogmatismos), el pragmatismo subyacente e inveterado del arbitraje internacional prevalecerá. 